\title{
Correction to: A Polyakov Formula for Sectors
}

\section{Clara L. Aldana ${ }^{1}$. Julie Rowlett ${ }^{2}$ (D)}

Published online: 25 November 2019

(c) The Author(s) 2019

\section{Correction to: J Geom Anal (2018) 28:1773-1839 https://doi.org/10.1007/s12220-017-9888-y}

Let $S_{\alpha}$ denote a finite circular sector of opening angle $\alpha \in(0, \pi)$ and radius one, and let $e^{-t \Delta_{\alpha}}$ denote the heat operator associated to the Dirichlet extension of the Laplacian. Based on recent joint work [2] and [3], we discovered an extra contribution to the variational Polyakov formula in [1] coming from the curved boundary component of the sector. Theorems 3 and 4 of [1] should have an added term $+\frac{1}{4 \pi}$. This calculation will appear in [2]. The corrected statements of these theorems are given below.

Theorem 1 (Theorem $3[1]$ ) Let $S_{\pi / 2} \subset \mathbb{R}^{2}$ be a circular sector of opening angle $\pi / 2$ and radius one. Then the variational Polyakov formula is

$$
\frac{\partial}{\partial \gamma}\left(-\left.\log \left(\operatorname{det}\left(\Delta_{S_{\gamma}}\right)\right)\right|_{\gamma=\pi / 2}=\frac{-\gamma_{e}}{4 \pi}+\frac{2}{3 \pi},\right.
$$

where $\gamma_{e}$ is the Euler-Mascheroni constant.

Theorem 2 (Theorem 4 [1]) Let $0<\alpha<\pi$, and let

$$
k_{\min }=\left\lceil\frac{-\pi}{2 \alpha}\right\rceil \text {, and } k_{\max }=\left\lfloor\frac{\pi}{2 \alpha}\right\rfloor \text { if } \frac{\pi}{2 \alpha} \notin \mathbb{Z} \text {, otherwise } k_{\max }=\frac{\pi}{2 \alpha}-1 \text {, }
$$

\section{The original article can be found online at https://doi.org/10.1007/s12220-017-9888-y.}

Julie Rowlett

julie.rowlett@chalmers.se

Clara L. Aldana

clara.aldana@uni.lu

1 Mathematics Research Unit, University of Luxembourg, 6, avenue de la Fonte, 4364 Esch-sur-Alzette, Luxembourg

2 Department of Mathematics, Chalmers University of Technology and the University of Gothenburg, 41296 Göteborg, Sweden 


$$
\begin{aligned}
& \text { and } W_{\alpha}=\left\{k \in\left(\mathbb{Z} \bigcap\left[k_{\text {min }}, k_{\text {max }}\right]\right) \backslash\left\{\frac{\ell \pi}{\alpha}\right\}_{\ell \in \mathbb{Z}}\right\} . \text { Then } \\
& \qquad \begin{aligned}
\mathcal{S}(\alpha): & =\left.\frac{\partial}{\partial \gamma}\left(-\log \left(\operatorname{det}\left(\Delta_{\gamma}\right)\right)\right)\right|_{\gamma=\alpha}=\frac{1}{3 \pi}+\frac{\pi}{12 \alpha^{2}} \\
& +\sum_{k \in W_{\alpha}} \frac{-2 \gamma_{e}+\log (2)-\log (1-\cos (2 k \alpha))}{4 \pi(1-\cos (2 k \alpha))} \\
& -\left(1-\delta_{\alpha, \frac{\pi}{n}}\right) \frac{2}{\alpha} \sin \left(\pi^{2} / \alpha\right) \int_{-\infty}^{\infty} \frac{\gamma_{e}+\log (2)-\log (1+\cosh (s))}{16 \pi(1+\cosh (s))\left(\cosh (\pi s / \alpha)-\cos \left(\pi^{2} / \alpha\right)\right)} d s,
\end{aligned}
\end{aligned}
$$

where $n \in \mathbb{N}$ is arbitrary and $\delta_{\alpha, \frac{\pi}{n}}$ denotes the Kronecker delta.

It therefore follows that the list of examples given following Theorem 4 in [1] should be revised accordingly:

(1) $\alpha=\frac{\pi}{4}, W_{\frac{\pi}{4}}=\{-2, \pm 1\},, \mathcal{S}\left(\frac{\pi}{4}\right)=\frac{-5 \gamma_{e}}{4 \pi}+\frac{\log (2)}{2 \pi}+\frac{5}{3 \pi} \sim 0.411167$

(2) $\alpha=\frac{\pi}{3}, W_{\frac{\pi}{3}}=\{-1,1\}, \mathcal{S}\left(\frac{\pi}{3}\right)=\frac{13}{12 \pi}-\frac{2 \gamma_{e}}{3 \pi}+\frac{\log (4 / 3)}{3 \pi} \sim 0.252871$

(3) $\alpha=\frac{\pi}{2}, W_{\frac{\pi}{2}}=\{-1\}, \mathcal{S}\left(\frac{\pi}{2}\right)=\frac{-\gamma_{e}}{4 \pi}+\frac{2}{3 \pi} \sim 0.166273$.

(4) For $\alpha \in] \frac{\pi}{2}, \pi\left[, W_{\alpha}=\emptyset\right.$, but $\sin \left(\pi^{2} / \alpha\right) \neq 0$. If $\alpha=\frac{2 \pi}{3}$, the integral converges rapidly, and a numerical computation gives an approximate value of 0.0075015 . Hence $\mathcal{S}\left(\frac{2 \pi}{3}\right) \sim \frac{1}{3 \pi}+\frac{3}{16 \pi}+\frac{3}{\pi}(0.0075015) \sim 0.1729498$.

\subsection{Misprint}

There is a two missing in Equation (1.3) of [1]. That equation should be:

$$
\partial_{t} \log \operatorname{det}\left(\Delta_{g_{t}}\right)=-\frac{1}{12 \pi} \int_{M} \sigma^{\prime}(t) \operatorname{Scal}_{t} d A_{g_{t}}+\partial_{t} \log \operatorname{Area}\left(M, g_{t}\right)
$$

Acknowledgements We thank Klaus Kirsten and Alexander Strohmaier who separately helped us find the mistake in our formula. The first author acknowledges the hospitality of the Max Planck Institute for Mathematics in Bonn during a visit in when then missing term was found. The second author is supported by the National Science Foundation Grant DMS-1440140 during the fall 2019 semester at MSRI and by the Swedish Research Council Grant 2018-03873.

\section{References}

1. Aldana, C.L., Rowlett, J.: A Polyakov formula for sectors. J. Geom. Anal. 28(2), 1773-1839 (2018)

2. Aldana, C.L., Kirsten, K., Rowlett, J.: A Polyakov formula for surfaces with conical singularities and boundary, pre-print

3. Nursultanov, M., Rowlett, J., Sher, D.: The heat kernel on curvilinear polygonal domains in surfaces, arXiv: 1905.00259

Publisher's Note Springer Nature remains neutral with regard to jurisdictional claims in published maps and institutional affiliations. 